\title{
FLOW CAN MAKE YOU RUN: EFFECTS OF OPTIC FLOW ON THE OVERGROUND WALK-TO-RUN TRANSITION
}

\author{
De Smet K., Malcolm P., Lenoir M., Aerts P., Segers V. and De Clercq, D. \\ University of Ghent (Belgium), Department of Movement and Sport Science, \\ e-mail: kristof.desmet@ugent.be
}

\section{INTRODUCTION}

Information from the visual system plays an important role in the regulation of locomotion. Perturbations of optic flow can have a powerful influence on whole body velocity in humans [1]. Mohler et al [2] indicated in a ramped protocol that mismatching treadmill speed and perceived speed by optic flow (by means of virtual reality) can evoke changes in walk-to-run transition speed. However, as perceiving an optic flow, which is related to walking speed, is very unusual (non familiar) on treadmill, it is necessary to confirm these findings in an ecological overground experiment in order to accept optic flow as a determinant of the WRT. Therefore, an overground experiment was conducted to investigate whether subjects, who experience an optic flow which is faster or slower then their actual walking speed, will change from walking to running at a respectively lower/higher transition speed.

\section{METHODS}

The experiment took place in a hallway $(1.8 \mathrm{~m}$ wide, $28 \mathrm{~m}$ long and $2.25 \mathrm{~m}$ high) (Figure 1). 11 female subjects were asked to start walking, from a stand still position, with a free chosen acceleration and make the walk-to-run transition (WRT).

Different visual conditions were created by rear-projecting black and white stripes $(20 \mathrm{~cm})$ on the sidewalls. Four conditions were tested. The control condition $\mathrm{C}$, in which stripes were not moving, was used as the reference condition. In the forward condition $\mathrm{F}$, stripes moved in the same direction as the subject at $+1 \mathrm{~m} . \mathrm{s}^{-1}$. In the backward condition $\mathrm{B}$, stripes moved in the opposite direction as the subjects at $-1 \mathrm{~m} \cdot \mathrm{s}^{-1}$. The double backward condition B2, where stripes moved in the opposite direction as the subject at $-2 \mathrm{~m} . \mathrm{s}^{-1}$, was used to check the dose-response relationship between optic flow and transition speed.

A synchronized measurement of subjects' speed (Noptel ${ }^{\circledR}$ Distance Laser $1000 \mathrm{~Hz}$ ) and foot contacts (Footscan ${ }^{\circledR}$ insoles $100 \mathrm{~Hz}$ ) permitted to analyse the speed of the transition step, which was the first step with a flight phase.

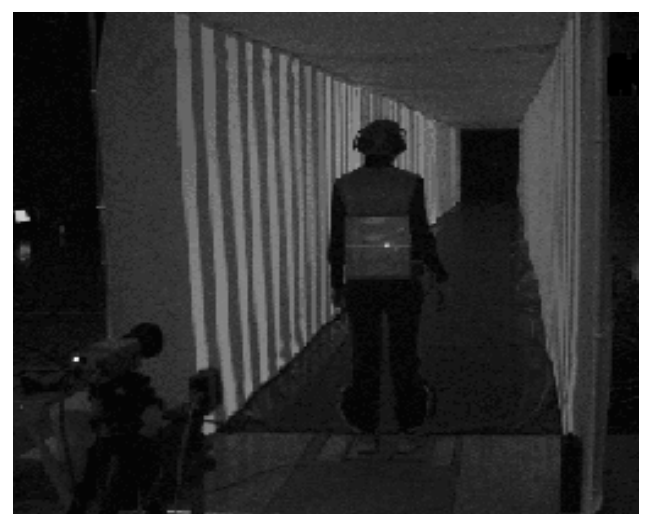

Figure 1: Subject ready to start in the hallway for control condition $\mathrm{C}$ (non-moving stripes). The reflecting cardboard was used to receive accurate data from the distance laser.

\section{RESULTS AND DISCUSSION}

Inter-trial variability for WRT-speed was low for each subject (ICCs> 0.822).

A repeated measures ANOVA compared the transition speed in the conditions. A significant main effect for visual condition was obtained on the WRT-speed $(\mathrm{F}=2.955, \mathrm{p}<.05)$. Table 1 shows the decrease in the WRT-speed from the $+1 \mathrm{~m} . \mathrm{s}^{-1}$ condition $\mathrm{F}$ to the $-2 \mathrm{~m} \cdot \mathrm{s}^{-1}$ condition B2. This indicates that subjects who get the impression of walking faster then they actually were, start to run at a lower speed and vice versa. Moreover, as visual perturbations became more powerful, differences in WRT-speed increased. This was indicated by the lower transition speed for the $-2 \mathrm{~m} . \mathrm{s}^{-1}$ backward double condition B2 compared to the $-1 \mathrm{~m} . \mathrm{s}^{-1}$ backward condition B.

Table 1: WRT-speed \pm standard deviations in four visual conditions.

\begin{tabular}{lcc}
\hline & WRT-speed $\left(\mathrm{m.s}^{-1}\right)$ & s.d. \\
\hline Forward & 2.634 & 0.205 \\
Control & 2.620 & 0.223 \\
Backward & 2.617 & 0.203 \\
Backward double & 2.572 & 0.236 \\
\hline
\end{tabular}

Optic flow (and with this the perceived speed) appears as a determinant of WRT-speed. These results confirm the data found on treadmill by Mohler et al [2]. Although previous research has enlightened other possible determinants that could trigger the WRT-speed [3], the findings in the current study indicate that modifications of the optic flow are able to override information from other sensor modalities during a transition sequence.

\section{CONCLUSIONS}

In summary, the protocol used in this investigation allowed to analyse the transition sequence in an ecological environment, where subjects were able to accelerate in the most spontaneous way. When optic flow was modified, subjects changed their WRT-speed. From this, we suggest that optic flow has a proprioceptive function in locomotor program control.

\section{REFERENCES}

1. Prokop T, et al., Exp Brain Res 114, 63-70, 1997.

2. Mohler BJ et al., Proceedings of the $1^{\text {st }}$ ACM SIGGRAPH, Los Angeles, California USA, 19-22, 2004.

3. Segers V, et al. Gait Posture, in press.

\section{ACKNOWLEDGEMENTS}

This research was supported by BOF-UGent B/06656/01-IV1. 